Journal of Case Reports 2020;10(1):25-28

\title{
IgG4-related Orbital Disease
}

\author{
Yu-Fang Huang ${ }^{1}$, Li-Chen Wei ${ }^{1}$, Hung-Chieh Chen ${ }^{2}$, Chih-Ying Wu ${ }^{3}$, Lu-Jen Chen ${ }^{3}$ \\ Departments of ${ }^{1}$ Ophthalmology, ${ }^{2}$ Radiology, ${ }^{3}$ Pathology, Taichung Veterans General Hospital, Taichung, Taiwan.
}

\section{Corresponding Author:}

Dr. Li-Chen Wei

Email: lichen5883@yahoo.com.tw

This is an Open Access article distributed under the terms of the Creative Commons Attribution License (creativecommons.org/ licenses/by/3.0).

Received Accepted

Published

June 19, 2019

January 2, 2020

February 10, 2020

\begin{abstract}
Case Report: A healthy 59-year-old male visited our hospital due to progressive bilateral temporal upper eyelid swelling for two years. On physical examination, elastic masses were palpable over temporal orbit without tenderness. The best corrected visual acuity of bilateral eyes was 6/6.7 on Snellen test. Intraocular pressure of right and left eye was $19 \mathrm{mmHg}$ and $22 \mathrm{mmHg}$ respectively. Extraocular movement revealed bilateral up gaze limitation and lateral gaze limitation of left eye. The computed tomography reported enlargement of bilateral lacrimal gland and left infraorbital nerve. Pathology diagnosis was compatible with IgG4-related orbital disease. The serum IgG4 level was $661 \mathrm{mg} /$ $\mathrm{dL}$. The patient received prednisolone $25 \mathrm{mg}$ twice daily initially and tapered gradually. The mass decreased in size and also extraocular movement improved after steroid use. Conclusion: We conclude that orbital IgG4-related disease is a special entity of orbital inflammation. Systemic steroid use is an effective treatment.
\end{abstract}

Keywords: Immunoglobulin G, Lacrimal Apparatus, Ocular Tonometry, Orbit, Visual Acquity.

\section{Introduction}

Idiopathic orbital inflammation had been found similar with autoimmune pancreatitis. Both diseases act like neoplasm and are characterized by variable degrees of fibrosis and chronic inflammation. Autoimmune pancreatitis has been identified as part of the spectrum of IgG4-associated systemic disease later. These are systemic syndromes characterized by elevated serum levels of IgG4 and IgG4-positive lymphoplasmacytic infiltrative lesions in the body. Orbital tissues are affected by IgG4-related conditions, too. It was first observed that Mikulicz's disease correlated with IgG4-related disease and later determined that IgG4-related disease can occur in any ocular adnexal tissues [1]. Here we report a IgG4-related orbital disease with enlargement of bilateral lacrimal gland.

\section{Case Report}

A 59-year-old male was generally healthy before visiting our clinic. He suffered from bilateral orbital mass over temporal upper area for more than one year. The mass was elastic, non-tender and progressively increasing in size. The Snellen best corrected visual acuity of bilateral eyes was 6/6.7. The intraocular pressure of right and left eye was $19 \mathrm{mmHg}$ and $22 \mathrm{mmHg}$ respectively. Up gaze limitation was noticed on bilateral eye movement and lateral limitation of left eye was his complaint [Fig.1].

He received contrast computed tomography, and enlargement of bilateral lacrimal gland and another enhancing left infra-orbital nerve were noted [Fig.2]. We further checked erythrocyte sedimentation rate (ESR), C-reactive protein (CRP) and eosinophil count which were all within normal range. The patient decided to receive biopsy. The pathology reported residual gland with dense plasma cells and lymphocytes infiltration with lymphoid follicles formation [Fig.3a]. The background was fibrotic with storiform fibrosis [Fig.3b]. Elevated IgG4/IgG ratio was above 40\% 
[Fig3c]. More than 100 IgG4-positive plasma cells per high power field were found [Fig.3d]. The findings were compatible with IgG4-related orbital disease. The serum IgG4 level of the patient was 661 $\mathrm{mg} / \mathrm{dL}$. He then received prednisolone $25 \mathrm{mg}$ twice daily and tapered gradually. The mass decreased in size and also extraocular movement improved after steroid use [Fig.4]. The orbital magnetic resonance imaging two months after prednisolone treatment revealed hypodense lesion over bilateral temporal upper orbit and left infra-orbital nerve, besides, hypergenecity noticed on contrast series. The lesion size decreased compared to previous CT images [Fig.5]. The most recent serum IgG-4 level was $246 \mathrm{mg} / \mathrm{dL}$.

\section{Discussion}

IgG4-related disease is initially described in autoimmune pancreatitis. The disease is characterized by tumefactive lesions at multiple sites, IgG4-positive lymphoplasmacytic infiltrate and fibrosis of the involved organ and often elevated serum IgG4 concentration. This condition has now been described in virtually every organ system: biliary tree, salivary glands, periorbital tissues, kidneys, lungs, lymph nodes, meninges,

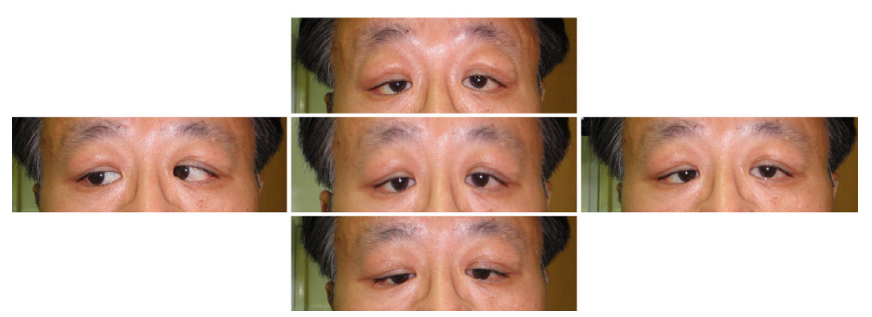

Fig.1: Extra-ocular movement showed bilateral eye up gaze limitation and left eye lateral gaze limitation before treatment.

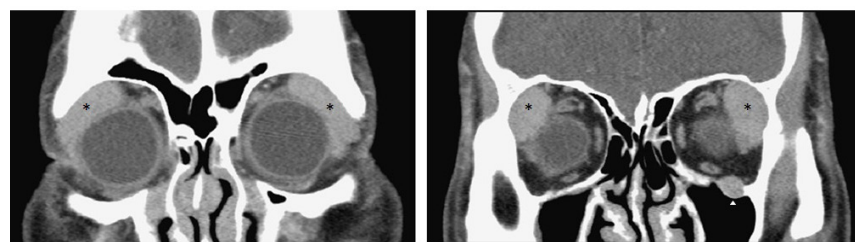

Fig.2: Computed tomography revealed bilateral lacrimal gland enlargement (asterisk) and enhancing lesion of left infra-orbital nerve (white arrow head). aorta, breast, prostate, thyroid, pericardium, and skin [1-7].

The three major histopathological features associated with IgG4-related disease are dense lymphoplasmacytic infiltrate; fibrosis, arranged

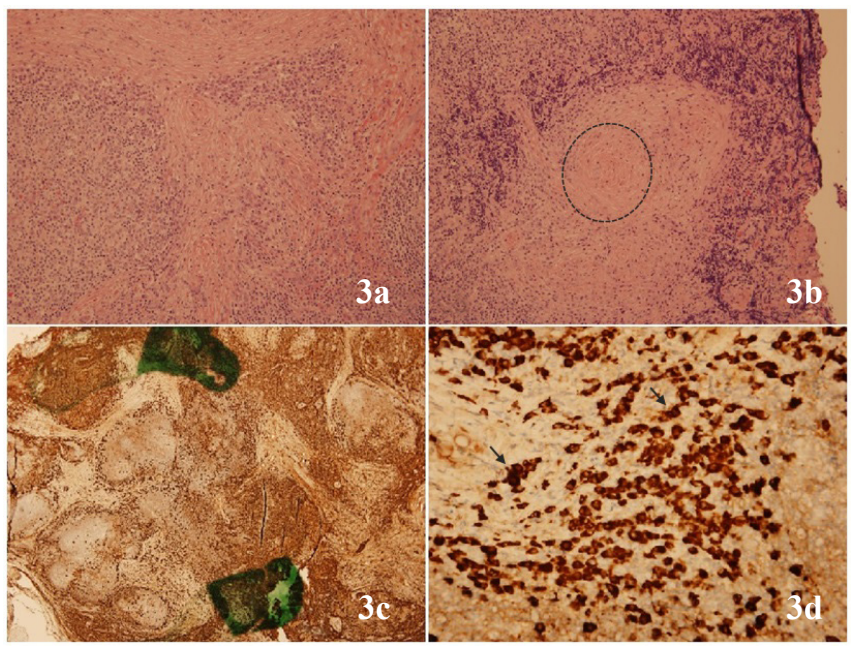

Fig.3: The pathology finding from tissue biopsy. (a): 200X, $H \& E$ stain showed dense plasma cells and lymphocytes infiltration. (b): 200X, H\&E stain showed storiform fibrosis (circled area). (c): 40X, IgG4 immunostaining, much IgG4 positive plasma cell noticed and the IgG4/IgG ratio is higher than 40\%. (d): 400X, IgG4 immunostaining, more than 100 IgG4 positive plasma cells (arrows) noticed under high power field.

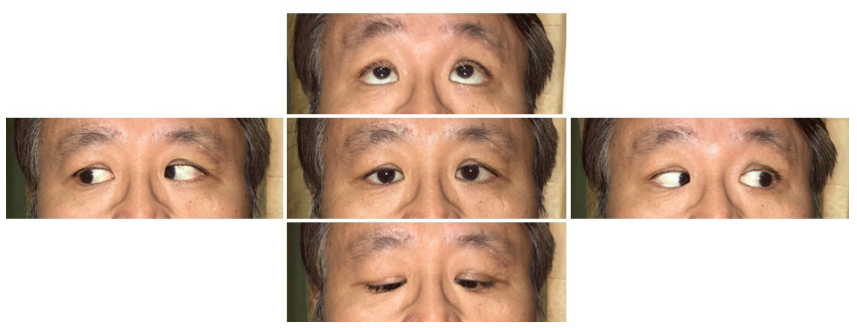

Fig.4: Improved extraocular movement and smaller bilateral temporal upper eyelid mass after steroid treatment.
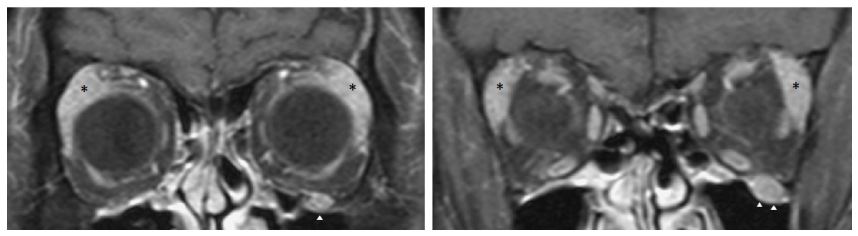

Fig.5: MRI revealed decreasing size in bilateral lacrimal gland (asterisk) and no obvious differences in left infraorbital nerve (arrow head) . 
at least focally in a storiform pattern; obliterative phlebitis. Other histopathological features associated with IgG4-related disease are phlebitis without obliteration of the lumen and increased numbers of eosinophils. The head and neck region is involved in approximately $20 \%$ patients with IgG4-related disease. Among patients with head and neck involvement, the orbit is affected in approximately $40 \%$ of cases [6]. In IgG4-related orbital disease, the inflammation mostly showed infiltrative lesions in ocular adnexa such as lacrimal glands, extraocular muscles, infraorbital nerves, optic nerve sheath, lacrimal sac and even cavernous sinus. IgG4-related orbital disease tends to affect people in middle-aged to elderly years. The prevalence in both genders is equal. On history taking, many patients mentioned about history of asthma as well as allergic rhinitis. The signs and symptoms are chronic eyelid swelling and proptosis. Ocular motility restriction was noticed in some patients. The periorbital pain or visual disturbance was generally not reported $[1,2,6,7,8]$.

WK Yu et al. reported a case series of 11 patients with ocular adnexal IgG4-related disease and $72.7 \%$ had lacrimal gland involvement, followed by orbit, extra-ocular muscles and eyelids [2]. C.A. Tiegs-Heiden et al. reviewed image findings of 27 patients diagnosed with IgG4-related orbital disease. Extra-ocular muscles were enlarged in $89 \%$ patients, most bilaterally. The lateral rectus was the most enlarged muscle. In $96 \%$ patients, the tendons of the extraocular muscles were spared. $70 \%$ patients had lacrimal gland enlargement. $44 \%$ patients had an infiltrative process within the orbital fat. Infra-orbital nerve enlargement was seen in $30 \%$ patients [9]. In patients with extraocular muscle enlargement, particularly when the tendons are spared and the lateral rectus is the most enlarged, IgG4-related disease should be a leading differential consideration.

In our case, the CT and MRI both showed bilateral lacrimal gland and left infraorbital nerve involvement. Typical radiologic features that have been reported for IgG4-related disease include soft tissue attenuation on computed tomography, hypointensity on T2-weighted magnetic resonance imaging owing to their increased cellularity and amount of fibrosis. The lesion also showed a homogeneous and gradual enhancement pattern, absence of vascular occlusion or compression, and presence of bone remodeling without destruction $[10,11]$.

The Japanese study group for IgG4-related ophthalmic disease had updated the diagnostic criteria in 2014 as following, (i) imaging shows enlargement of the lacrimal gland, trigeminal nerve, or extra-ocular muscle as well as masses, enlargement, or hypertrophic lesions in various ophthalmic tissues; (ii) histopathologic examination shows marked lymphocyte and plasmacyte infiltration, and sometimes fibrosis. A germinal center is frequently observed. IgG4+ plasmacytes are found and satisfy the following criteria: ratio of IgG4 + cells to $\mathrm{IgG}+$ cells of $40 \%$ or above, or more than 50 IgG4+ cells per high-power field $(\times 400)$ [3]. Blood test shows elevated serum IgG4 (>135 $\mathrm{mg} / \mathrm{dL}$ ) [3]. The patient in our case meets all three criteria. The diagnosis of IgG4-related ophthalmic disease is definite. The Japanese experts suggested the rate of IgG4-related orbital disease in orbital lymphoproliferative disease was $26 \%$ to $33 \%$ [12]. For optimal treatment, IgG4 related orbital disease must be differentiated from the following: orbital pseudotumor, idiopathic orbital myositis, marginal zone B-cell lymphoma, anti-neutrophil cytoplasmic antibody-mediated systemic vasculitis, and reactive lymphoid hyperplasia without IgG4positive plasma cell [1]. Orbital pseudotumor classically present with orbital pain and orbital myositis characteristically causes enlargement of the entire muscle, including the tendon. The treatment of orbital IgG4-related diseases may include systemic steroids, radiotherapy, or rituximab. Glucocorticoids are typically the first 
line of therapy. Some suggested treating patients initially with prednisolone at a dose of $0.6 \mathrm{mg}$ per kilogram of body weight per day for 2 to 4 weeks. Slowly taper within 3 months or even keep low dose upto 3 years were both mentioned. Azathioprine, mycophenolate mofetil, and methotrexate are used frequently as glucocorticoid-sparing agents or remission-maintenance drugs after glucocorticoidinduced remissions. In refractory or recurrent disease, B-cell depletion with rituximab appears to be useful. WK Yu had found that patients with lower serum $\mathrm{IgG} 4$ and $\mathrm{IgG} 4 / \mathrm{IgG}$ ratio at diagnosis were associated with poor response [2]. Baseline concentration of serum IgG4, IgE and eosinophil count were found to be independently predictive relapse risk following treatment [1-7]. In our case, the patient responded well to oral prednisolone. The initially blood test revealed elevated serum IgG4 and normal $\operatorname{IgE}$ and eosinopohil count. However, long-term follow up is necessary for recurrence surveillance.

\section{Conclusion}

IgG4-related orbital disease should be differentiated from orbital tumor or other inflammatory disease. Biopsy provides definite diagnosis with unique presentation. Oral prednisolone is the choice of treatment and usually effective. We reported a case of orbital IgG4-related disease in our institute.

Contributors: YFH: manuscript editing, literature review; LCW: manuscript writing, and patient management; HCC: critical inputs into the manuscript and imaging; CYW, LJC: literature review, discussion and histopathology. LCW will act as a study guarantor. All authors approved the final version of this manuscript and are responsible for all aspects of the study.

Funding: None; Competing interests: None stated.

\section{References}

1. Toshinobu K, Suzuko M. Orbital IgG4-related disease: clinical features and diagnosis. ISRN Rheumatology. 2012;412896.

2. Yu WK, Kao SC, Yang CF, Lee FL, Tsai CC. Ocular adnexal IgG4-related disease: clinical features, outcome, and factors associated with response to systemic steroids. Jpn J Ophthalmol. 2015;59:8-13.

3. Goto H1, Takahira M, Azumi A. Diagnostic criteria for IgG4-related ophthalmic disease. Jpn J Ophthalmol. 2015;59:1-7.

4. Deshpande V, Zen Y, Chan JKC, Yi EE, Sato Y, Yoshino T, Klöppel G, et al. Consensus statement on the pathology of IgG4-related disease. Mod Pathol. 2012;25:11811192.

5. John HS, Yoh Z, Vikram D. IgG4-related disease. N Engl J Med. 2012;366:539-551.

6. Cecilia SL, George JH, Courtney LK, Aaron YL, Gregory PVS, Steven MC, et al. IgG4-associated orbital and ocular inflammation. J Ophthalmic Inflamm Infect. 2015;5:15.

7. Jose' AP, James AG, Ahmet D, Anuradha A, Thomas EW, Diva RS. Orbital inflammation with IgG4-positive plasma cells. Arch ophthalmol. 2011;129:421-428.

8. Japanese study group of IgG4-related ophthalmic disease. A prevalence study of IgG4-related ophthalmic disease in Japan. Jpn J Ophthalmol. 2013;57:573-579.

9. Tiegs-Heiden CA, Eckel LJ, Hunt CH, Diwhn FE, Schwartz KM, Kallmes DF, et al. Immunoglobulin G4-related disease of the orbit: imaging features in 27 patients. AJNR Am J Neuroradiol. 2014;35:1393-1397.

10. Daniel T, Suzanne KF, David K, Grove A, Flay A, Cunnane $\mathrm{M}$, et al. Radiographic patterns of orbital involvement in IgG4-related disease. Ophthal Plast Reconstr Surg. 2013;29:261-266.

11. Akifumi F, Osamu S, Margaret NC, Hideharu S. IgG4related disease of the head and neck: CT and MR imaging manifestations. Radiographics. 2012;32:1945-1958.

12. Takahira M, Ozawa Y, Kawano M, Zen Y, Hamaoka $\mathrm{S}$, Yamada $\mathrm{K}$, et al. Clinical aspects of IgG4-related orbital inflammation in a case series of ocular adnexal lymphoproliferative disorders. Int $\mathrm{J}$ Rheumatol. 2012;635473. 